\title{
Étude de l'impact des variabilités climatiques sur les ressources hydriques d'un milieu tropical humide
} Cas du département de Bongouanou (Est de la Côte d'ivoire)

\section{Impact of climatic variability on water resources in a humid tropical climate}

\author{
Case of Bongouanou department (eastern Ivory Coast)
}

\author{
Emile Assie Assemian, Fernand Koffi Kouame, Éric Valère Djagoua, Kouadio \\ Affian, Jean Patrice Roger Jourda, Miessan Adja, Théophile Lasm et Jean Biemi
}

Volume 26, numéro 3, 2013

Reçu le 13 mars 2013, accepté le 2 juillet 2013

URI : https://id.erudit.org/iderudit/1018789ar

DOI : https://doi.org/10.7202/1018789ar

Aller au sommaire du numéro

Éditeur(s)

Université du Québec - INRS-Eau, Terre et Environnement (INRS-ETE)

ISSN

1718-8598 (numérique)

Découvrir la revue

Citer cet article

Assemian, E. A., Kouame, F. K., Djagoua, É. V., Affian, K., Jourda, J., Adja, M., Lasm, T. \& Biemi, J. (2013). Étude de l'impact des variabilités climatiques sur les ressources hydriques d'un milieu tropical humide : cas du département de Bongouanou (Est de la Côte d'ivoire). Revue des sciences de l'eau / Journal of Water Science, 26(3), 247-261. https://doi.org/10.7202/1018789ar
Résumé de l'article

Cette étude vise à montrer l'apparition d'un changement climatique dans ces dernières décennies dans le département de Bongouanou, situé à l'est de la Côte d'Ivoire (milieu tropical humide) et à élucider son impact sur l'alimentation des ressources en eau souterraine. Pour ce faire, diverses méthodes statistiques et hydrologiques ont été utilisées. L'application de l'indice de Nicholson et le test de Pettitt à la série pluviométrique (1920-2005) a mis en évidence une variabilité climatique, caractérisée par deux grandes phases différentes. Les résultats montrent une rupture en 1968 qui marque une modification des régimes pluviométriques et hydrologiques. Cette rupture s'accompagne d'une diminution de la pluviométrie d'environ $19 \%$ en moyenne et une augmentation de $0,7^{\circ} \mathrm{C}$ de la température du milieu. Il s'agit donc d'un réchauffement climatique dans ce milieu tropical qui se caractérise par deux grandes périodes : une période humide de 1920 à 1968 et une période sèche ou déficitaire de 1968 à 2005. La méthode du bilan hydrique et celle de Maillet ont permis de mettre en évidence les impacts de ce réchauffement climatique sur les ressources en eau de la région. Les résultats montrent qu'avant la rupture de 1968, la recharge moyenne annuelle était estimée à $217 \mathrm{~mm}$; elle est passée à $145 \mathrm{~mm}$ en période déficitaire, soit une baisse d'environ $33 \%$. Le ruissellement moyen annuel qui était de $80 \mathrm{~mm}$, est réduit à $35 \mathrm{~mm}$. Les coefficients de tarissement calculés montrent une vidange rapide des réserves régulatrices en période sèche ( 21 jours) par rapport à la période humide (27 jours). Les volumes moyens d'eau mobilisés par les aquifères chaque année ont subi une baisse de $30 \%$. En période humide, ce volume était estimé à environ $1,475 \mathrm{~km}^{3}$ dans la région de Bongouanou. La concordance des résultats d'évaluation des recharges et des volumes d'eau mobilisés par ces deux approches indépendantes (bilan hydrique et analyse d'hydrogrammes) avant et après la rupture, est remarquable. Cela montre aussi l'importance de précipitations importantes et régulières pour la recharge efficace des aquifères de socle.
Ce document est protégé par la loi sur le droit d'auteur. L’utilisation des services d’Érudit (y compris la reproduction) est assujettie à sa politique d'utilisation que vous pouvez consulter en ligne.

https://apropos.erudit.org/fr/usagers/politique-dutilisation/ 


\section{ÉTUDE DE L'IMPACT DES VARIABILITÉS CLIMATIQUES SUR LES RESSOURCES HYDRIQUES D'UN MILIEU TROPICAL HUMIDE : CAS DU DÉPARTEMENT DE BONGOUANOU (EST DE LA CÔTE D'IVOIRE)}

Impact of climatic variability on water resources in a bumid tropical climate: case of Bongouanou department (eastern Ivory Coast)

Emile Assie ASSEMLAN*, Fernand Koffi KOUAME, Éric V ALÈre DJAGOUA, Kouadio AFFLAN, Jean Patrice Roger JOURDA, MIESSAN ADJA,THÉOPHILE LASM, JEAN BIEMI

UFR des Sciences de la Terre et des Ressources Minières, Université Félix Houphouet Boigny, Abidjan, BP 582, Abidjan 22, Côte d'Ivoire

Reçu le 13 mars 2013, accepté le 2 juillet 2013

\section{RÉSUMÉ}

Cette étude vise à montrer l'apparition d'un changement climatique dans ces dernières décennies dans le département de Bongouanou, situé à l'est de la Côte d'Ivoire (milieu tropical humide) et à élucider son impact sur l'alimentation des ressources en eau souterraine. Pour ce faire, diverses méthodes statistiques et hydrologiques ont été utilisées. L'application de l'indice de Nicholson et le test de Pettitt à la série pluviométrique (1920-2005) a mis en évidence une variabilité climatique, caractérisée par deux grandes phases différentes. Les résultats montrent une rupture en 1968 qui marque une modification des régimes pluviométriques et hydrologiques. Cette rupture s'accompagne d'une diminution de la pluviométrie d'environ $19 \%$ en moyenne et une augmentation de $0,7^{\circ} \mathrm{C}$ de la température du milieu. Il s'agit donc d'un réchauffement climatique dans ce milieu tropical qui se caractérise par deux grandes périodes : une période humide de 1920 à 1968 et une période sèche ou déficitaire de 1968 à 2005. La méthode du bilan hydrique et celle de Maillet ont permis de mettre en évidence les impacts de ce réchauffement climatique sur les ressources en eau de la région. Les résultats montrent qu'avant la rupture de 1968, la recharge moyenne annuelle était estimée à $217 \mathrm{~mm}$; elle est passée à $145 \mathrm{~mm}$ en période déficitaire, soit une baisse d'environ $33 \%$. Le ruissellement moyen annuel qui était de $80 \mathrm{~mm}$, est réduit à $35 \mathrm{~mm}$. Les coefficients de tarissement calculés montrent une vidange rapide des réserves régulatrices en période sèche ( 21 jours) par rapport à la période humide (27 jours). Les volumes moyens d'eau mobilisés par les aquiferes chaque année ont subi une baisse de $30 \%$. En période humide, ce volume était estimé à environ $1,475 \mathrm{~km}^{3}$ dans la région de Bongouanou. La concordance des résultats d'évaluation des recharges et des volumes d'eau mobilisés par ces deux approches indépendantes (bilan hydrique et analyse d'hydrogrammes) avant et après la rupture, est remarquable. Cela montre aussi l'importance de précipitations importantes et régulières pour la recharge efficace des aquiferes de socle.

\section{Mots clés : Variabilité climatique, eau souterraine, eau de surface, département de Bongouanou Côte d'Ivoire.}




\section{ABSTRACT}

This study aimed to show the appearance of a climatic change in the last decade in Bonguanou department, situated in eastern Ivory Coast (humid tropical zone) and to elucidate its impact on groundwater supply. To this end, different statistical and hydrologic methods have been used. The application of the Nicholson index and Pettitt test to the rainfall series (1920-2005) revealed climatic variability, characterized by two great different phases. The results show a rupture in 1968, which marks an alteration in the rainfall and hydrological regimes. This rupture is associated with a diminution of the rainfall by about $19 \%$ on average and an increase of $0.7^{\circ} \mathrm{C}$ in the temperature of the region. It suggests climatic warming in this tropical environment and is characterized by two great periods: a humid period, 1920 to 1968, and a dry or deficit period, 1969 to 2005. The hydrological balance method and the Maillet method were used to determine the impacts of climatic warming on water resources in this region. The results show that, before the rupture (1968), the annual average recharge was $217 \mathrm{~mm}$; in dry period, it decreased to $145 \mathrm{~mm}$, a drop of $33 \%$. The annual average surface flow, which was $80 \mathrm{~mm}$ before the rupture, is reduced to $35 \mathrm{~mm}$. The estimated recession coefficients show a more rapid draining of the regulating reserves in the dry period $(21 \mathrm{~d})$ compared with the humid period $(27 \mathrm{~d})$. The average annual mobilized water volumes in the aquifers have sustained a drop of $30 \%$. In humid period, this annual average volume was estimated to have been $1.475 \mathrm{~km}^{3}$ in the Bongouanou region. The agreement of the estimates for the recharge volume and for the mobilized water volumes, by two independent approaches (hydrological balance and hydrogram analysis), before and after the 1968 rupture, shows the importance of regular and sustained rainfall for recharge of the hard rock aquifer.

\section{Key-words: Climatic variability, groundwater, surface water, Bongouanou department, Ivory Coast}

\section{INTRODUCTION}

Les études portant sur l'impact des changements climatiques sur les ressources en eau ont intéressé la communauté mondiale suite à plusieurs manifestations climatiques de grande ampleur. Parmi celles-ci, on note la sécheresse qui a touchéles deux bandes tropicales de notre planète, surtout les pays sahéliens d'Afrique de l'Ouest, depuis les années 1970 (SIRCOULON, 1976). À cette sécheresse, s'ajoutent les constatations de l'Organisation Météorologique Mondiale (OMM) sur le réchauffement de la planète, supérieur à $0,7^{\circ} \mathrm{C}$, depuis le début du siècle dernier et les récents phénomènes d'El Niño (CANTAT, 1995 NICHOLLS, 1988). La réalité de la variabilité climatique, dont les causes sont difficiles à cerner, se manifeste dans certains pays par de longues périodes de sécheresse avec, pour conséquences, des effets négatifs sur le cycle hydrologique, l'environnement et les activités socio-économiques. La sécheresse qui a sévi depuis une trentaine d'années dans les pays sahéliens a connu une extension vers les pays humides en bordure du Golfe de Guinée, avec de graves conséquences : la baisse des précipitations, la baisse des niveaux piézométriques et la chute des débits des cours d'eau (SAVANÉ et al., 2001; SERVAT et al., 1998). Une tendance à la baisse de la pluviométrie a été observée en Afrique de l'Ouest à partir de la fin des années 1960 et au début des années 1971 (BRICQUET et al., 1997; HUBERT et al., 1987; MAHÉ et OLIVRY, 1995; SERVAT et al., 1998). Le déficit des apports a été estimé à $16 \%$ pour l'Afrique tropicale.

La Côte d'Ivoire n'est pas en marge de ce phénomène. En effet, des études sur la variabilité climatique ont montré qu'une tendance à la sécheresse s'est manifestée à partir de la fin de la décennie 1960 (BIGOT et al., 2005; BROU, 1997). Ce phénomène ne s'est pas réalisé de façon homogène dans le temps; il a d'abord affecté le nord puis progressivement s'est étendu vers le Centre et, enfin, sur le littoral (KOUASSI, 2008). Ces anomalies pluviométriques constatées depuis près de quatre décennies ont connu une résonance exceptionnelle dans les régions Nord et Centre du pays.

L'exemple de l'est de la Côte d'Ivoire choisi pour cette étude est intéressant car, cette région subit de plus en plus de longues saisons sèches chaque année au cours des dernières décennies. Elle est surtout marquée par de fortes pressions anthropiques. La population locale, principal acteur de cette pression sur le couvert végétal, est caractérisée par une forte densité de population $\left(30\right.$ habitants $\left.\bullet \mathrm{km}^{-2}\right)$ qui continue à croître. Les eaux souterraines, principales ressources d'approvisionnement en eau potable, semblent être affectées par les manifestations du changement climatique.

La caractérisation de la variabilité climatique et son influence sur les ressources en eaux souterraines à l'aide de méthodes statistiques et hydrologiques constituent les objectifs majeurs de cette étude. Il s'agit donc de mettre en évidence les périodes de ces changements climatiques et d'évaluer leurs impacts sur les ressources hydriques. Le secteur Est de la Côte d'Ivoire qui sera étudié est le département de Bongouanou, précisément compris entre les latitudes $6^{\circ} 9^{\prime} 50^{\prime \prime} \mathrm{N}$ et $6^{\circ} 59^{\prime} \mathrm{N}$ et les longitudes $3^{\circ} 44^{\prime} \mathrm{W}$ et $4^{\circ} 47^{\prime} \mathrm{W}$. Sur le plan administratif, il fait partie de la région du N'Zi-Comoé dont Dimbokro est le chef-lieu de région, et couvre une superficie de $6670 \mathrm{~km}^{2}$, soit 2 \% de la superficie de la Côte d'Ivoire. Le milieu hydrologique est composé de trois bassins versants de surface, à savoir le bassin du N'Zi à l'ouest et au nord, le bassin de la Comoé à l'est et le bassin d'Agnéby-M'bé au sud (Figure 1). La région bénéficie d'un climat tropical humide, avec une pluviométrie moyenne annuelle actuellement comprise entre 700 et $1500 \mathrm{~mm}$. Dans ce milieu, le sol est essentiellement hydromorphe et 


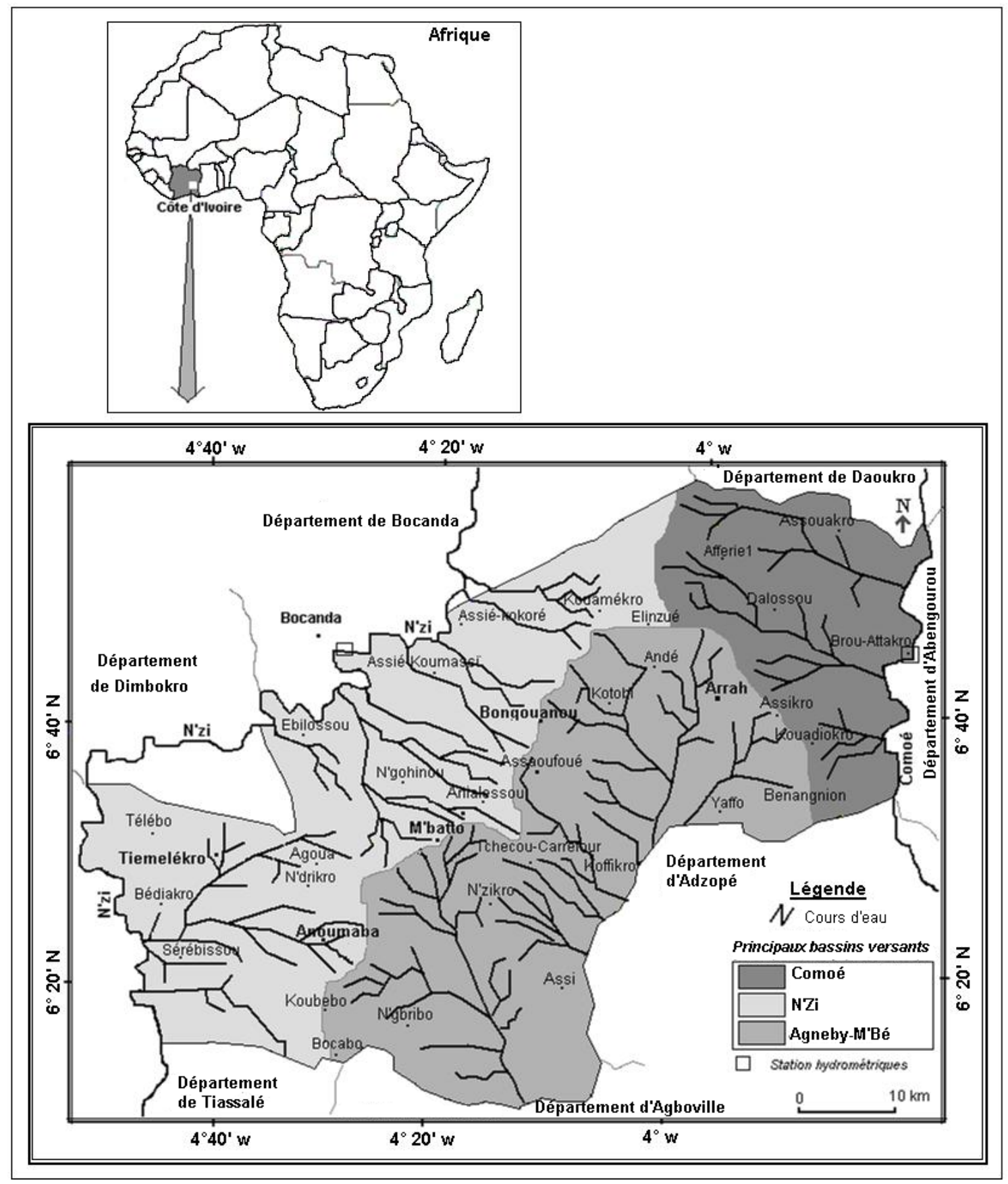

Figure 1. Situation géographique du département de Bongouanou. Geographic location of Bongouanou department.

ferrallitique et présente des textures et des structures favorables à l'infiltration des eaux de pluie. Le taux d'évapotranspiration dépend de l'état de saturation du sol et de son état de surface. Lorsque l'humidité du sol a dépassé le point de ressuyage, tout apport de pluie supplémentaire se retrouve dans l'écoulement superficiel et souterrain.

Le couvert végétal, très hétérogène, passe du fait de la déforestation, au début du $\mathrm{XX}^{\mathrm{e}}$ siècle, des forêts denses humides sempervirentes en accord avec une pluviométrie moyenne annuelle excédant $1600 \mathrm{~mm}$ (GUILLAUMET et
ADJANOHOUN, 1971; LENEUF, 1959), à la végétation actuelle de type jachère, moins ligneux, caractéristique des régions où la pluviométrie annuelle est inférieure à $1200 \mathrm{~mm}$. Au début des années 1950, la forêt dense primitive couvrait plus de 600000 ha, soit $81 \%$ de la superficie totale. En 1980, il n'en restait que 320000 ha dont 210000 ha de forêts classées (SODEFOR, 1992). Actuellement, cette forêt primitive n'existe pratiquement plus dans le département de Bongouanou à cause de la forte pression anthropique. Cette pression est liée à la pratique de l'exploitation du bois, à la culture itinérante sur brûlis, à la mobilisation de vastes territoires pour les cultures 
de rente et industrielles (cacao, café, hévéa et palmier à huile). Cette agression constante et irréversible de la forêt fait que les périodes de jachères deviennent de plus en plus courtes et, par conséquent, la reconstitution de l'écosystème forestier est de plus en plus difficile. Il naît alors un type de végétation nouveau formé d'espèces à croissance rapide (arbustes et herbes) à caractère savanicole, et on constate en parallèle la diminution générale de la biodiversité. La figure 2 issue de la classification supervisée par maximum de vraisemblance des compositions colorées des images ETM+ de Landsat7 du 13 avril 2003, met en évidence l'actuelle occupation du sol du département de Bongouanou. Le résultat de cette classification supervisée montre qu'il ne reste que 3253 ha de forêts primitives dans les zones classées. La déforestation et la dégradation des forêts entrainent très vraisemblablement un changement climatique qui, en retour, endommage les forêts (WWF, 2011). Leur destruction massive a donc certainement une incidence sur le climat local.
Le milieu géologique est en grande partie constitué de formations paléoprotérozoïques dont la lithologie est composée de métasiltites, de métaarénites, d'intrusions granitiques et de leurs produits d'altération (notamment, cuirasses latéritiques). On observe aussi de petites formations quaternaires en bordure des rivières N'Zi et Comoé, formées de sables lessivés (Figure 3). Les cuirasses bauxitiques épousent des topographies importantes, pouvant atteindre $600 \mathrm{~m}$ d'altitude. Les autres formations sont relativement plates et l'altitude moyenne est d'environ $120 \mathrm{~m}$. Le sous-sol est essentiellement formé d'aquiferes discontinus de socle (altérites et horizon fissuré) de nature schisteuse ou granitique. Les altérites ont des épaisseurs qui varient de quelques mètres à plus de $100 \mathrm{~m}$ et contiennent souvent des nappes d'eau importantes en relation avec le degré d'altération de la roche-mère. Ces systèmes d'aquifères discontinus sont, pour ce qui concerne leur recharge, sous l'influence des fluctuations saisonnières. Contrairement aux aquifères continus des bassins sédimentaires, leur perméabilité

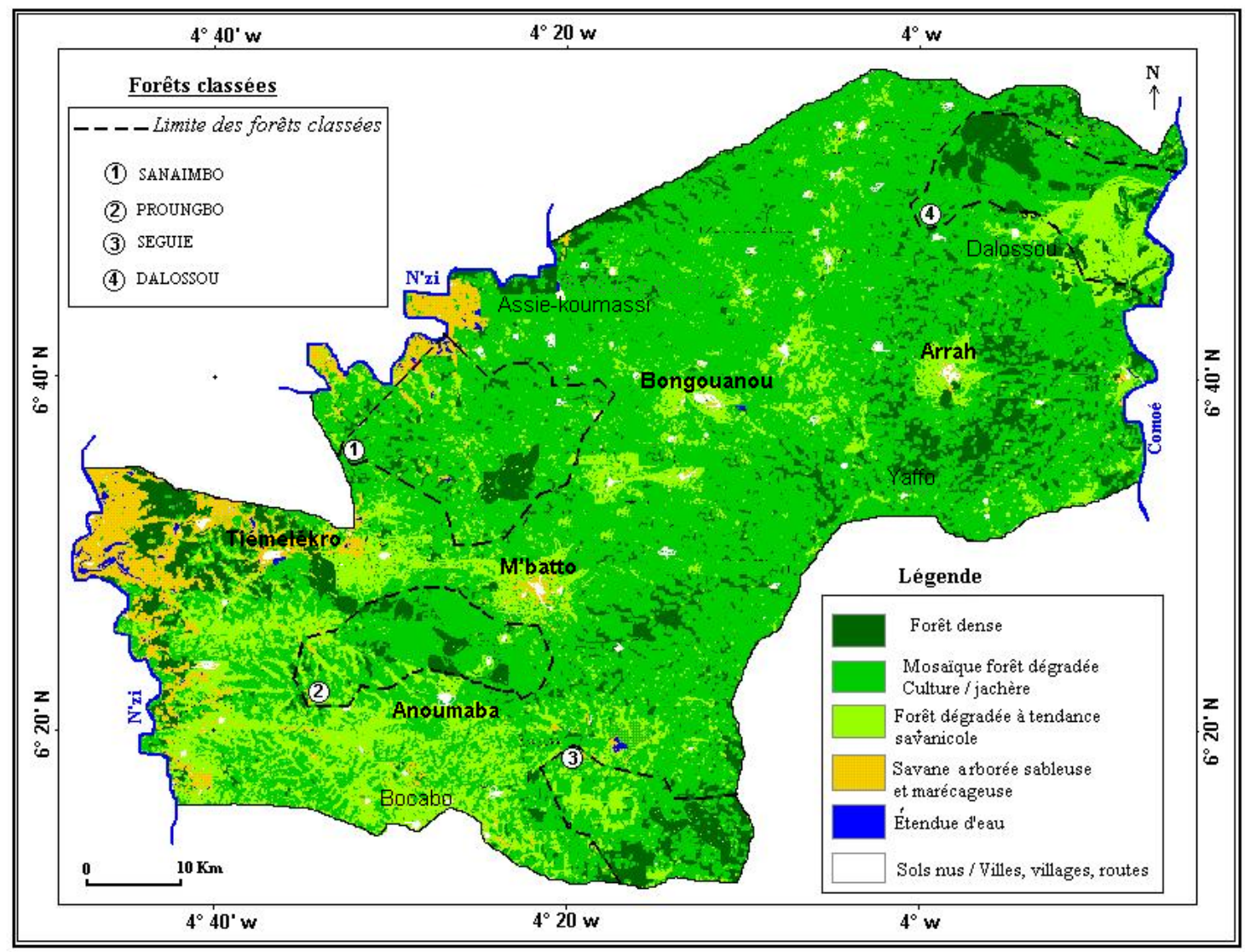

Figure 2. Carte d'occupation du sol du département de Bongouanou issue de la classification supervisée d'une composition colorée des images ETM+ de Landsat 7 (13-4-2003) montrant les restes des forêts primitives.

Map of land use in Bongouanou department obtained from the supervised classification of Landsat 7 ETM+(13-4-2003) colour composition showing the remainder of primitive forests. 


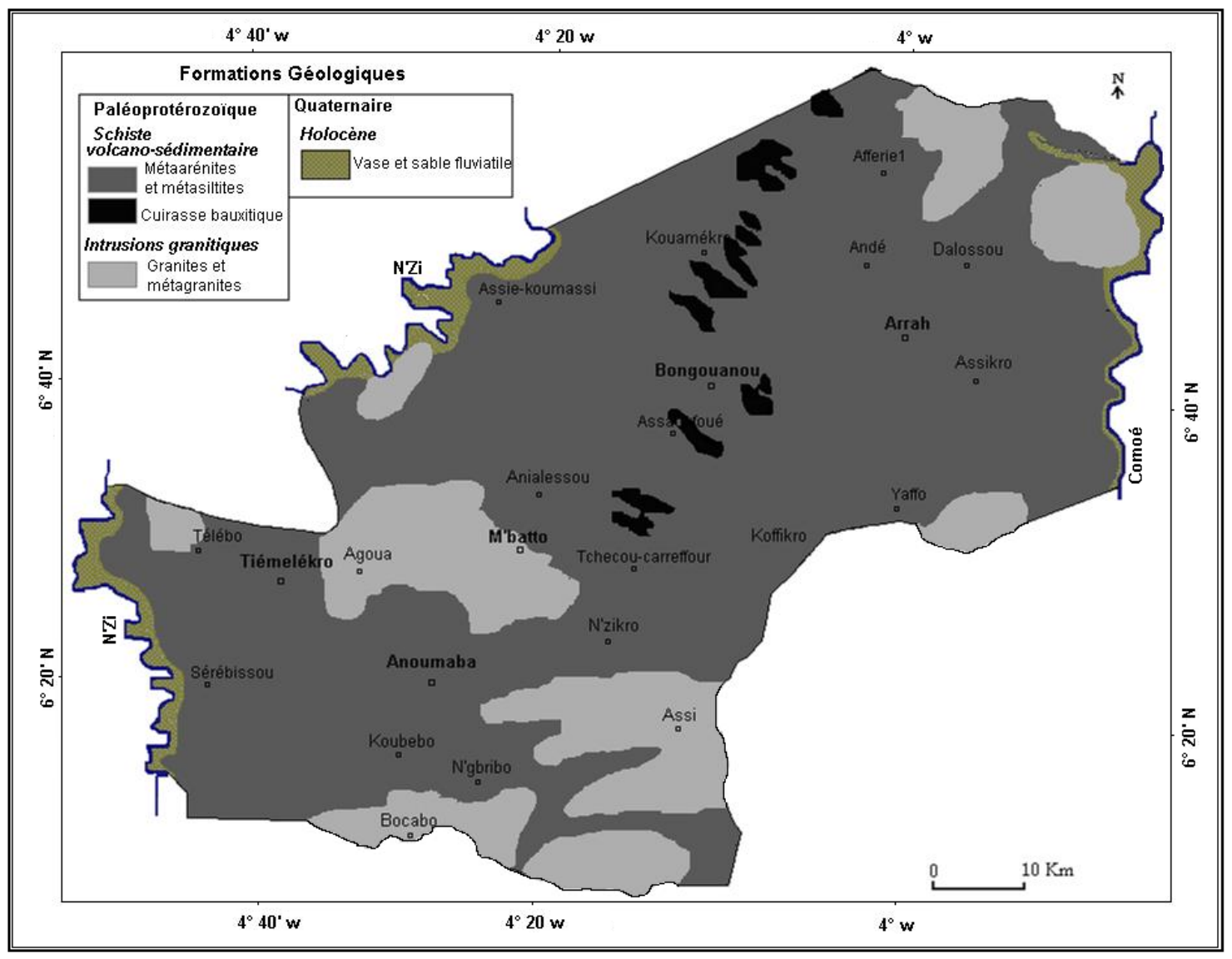

Figure 3. Carte géologique simplifiée du département de Bongouanou. Simplified geological map of Bongouanou department.

et porosité sont relativement faibles et leur productivité très modeste.

\section{MATÉRIEL ET MÉTHODES}

\subsection{Données}

Les données climatiques (1920-2005) de la région de Bongouanou ont été obtenues auprès de la société d'exploitation pour le développement aéroportuaire et météorologique de la Côte d'Ivoire (SODEXAM). Les données hydrométriques ont été mises à notre disposition par la direction de l'hydraulique humaine, précisément la sous-direction de l'hydrologie. Ces données concernent les débits journaliers de la rivière N'Zi et du fleuve Comoé, couvrant la période 1953 à 1987, recueillies respectivement aux stations hydrométriques près de Bocanda et de Brou-Attakro.

\subsection{Méthodes}

\subsubsection{Méthodes de mise en évidence du changement climatique}

La méthodologie de cette étude a commencé par une analyse statistique de la série pluviométrique. Ainsi, la moyenne, l'écarttype et le coefficient de variation ont été calculés. Le coefficient de variation $(\mathrm{CV})$, qui est le rapport entre l'écart-type et la moyenne, permet d'apprécier la dispersion de la pluviométrie autour de la moyenne.

Des tests de détection de ruptures dans une série pluviométrique ont été élaborés pour mettre en évidence un changement climatique au sein d'une période chronologique. On peut citer, entre autre, la procédure de segmentation de HUBERT (HUBERT et al., 1989) et le test de PETTITT (1979). En effet, une rupture est définie comme un changement dans la loi de probabilité des variables aléatoires dont les réalisations successives définissent les séries chronologiques étudiées (SERVAT et al., 1998). Dans cette étude, pour la détection des ruptures, nous avons appliqué le test de Pettitt. 
Il consiste à décomposer la série principale de $\mathrm{N}$ éléments en deux sous-séries à chaque instant $\mathrm{t}$ compris entre 1 et $\mathrm{N}-1$. La série principale présente une rupture à l'instant $\mathrm{t}$ si les deux sous-séries ont des distributions différentes. Les variables de Pettitt $(\mathrm{U})$ sont définies par l'équation 1 suivante :

$$
\mathrm{U}=\sum_{\substack{0 \leq i \leq m \\ 0<j<n}} \mathrm{D}(\mathrm{i}, \mathrm{j})
$$

où $\quad \mathrm{D}(\mathrm{i}, \mathrm{j})=\operatorname{sgn}\left(\mathrm{x}_{\mathrm{i}}-\mathrm{x}_{\mathrm{i}}\right)$

$\operatorname{sgn}(\mathrm{x})=1$ si $\mathrm{x}>0$

$\operatorname{sgn}(\mathrm{x})=0$ si $\mathrm{x}=0$

$\operatorname{sgn}(\mathrm{x})=-1$ si $\mathrm{x}<0$

La probabilité (Prob) de dépassement d'une valeur k est définie et permet d'apprécier l'importance de la rupture.

$\operatorname{Prob}(\mathrm{kn}>\mathrm{k}) \approx 2 \exp / \operatorname{Prob}(\mathrm{kn}>\mathrm{k}) \approx 2 \exp \left(-6 \mathrm{k}^{2} / \mathrm{n}^{3}+\mathrm{n}^{2}\right)$

L'absence de rupture dans la série de taille $\mathrm{N}$ constitue l'hypothèse nulle. Si l'hypothèse nulle est rejetée, une estimation de la date de la rupture est donnée à cet instant, définissant le maximum en valeur absolue de la variable $U$.

Lindice de NICHOLSON (1994) ou indice pluviométrique (Ip) est une variable centrée réduite qui traduit l'écart de la pluie d'une année i à la pluie moyenne de la période considéré par rapport à l'écart-type. Cet écart traduit soit des excédents, soit des déficits pluviométriques annuels de la série climatique. Ainsi, les successions des périodes excédentaires et déficitaires mettent en évidence les changements climatiques de cette série. L'expression mathématique de l'indice de Nicholson est définie par l'équation 2 :

$$
\mathrm{Ip}=\frac{\mathrm{Pi}-\mathrm{P}}{\sigma}
$$

Ip : indice de Nicholson ou Indice pluviométrie

$\mathrm{Pi}$ : pluie annelle de l'année i

$\mathrm{P}$ : moyenne pluviométrique de la période considérée

$\sigma$ : écart-type de cette série.

Par ailleurs, une confirmation des résultats de détection des changements pourrait être vérifiée par une comparaison des températures moyennes mensuelles de chaque période déterminée.

\subsubsection{Bilan hydrologique}

Il existe plusieurs méthodes permettant d'estimer les paramètres du bilan hydrologique. On peut citer, entre autre : la méthode de TURC (1931), de THORNTHWAITE (1948) et de PENMAN (1948). Chacune de ces méthodes a ses avantages et ses limites. Pour la détermination des paramètres du bilan hydrique, nous avons opté pour la méthode de Thornthwaite. Le choix de cette méthode s'explique par le manque de données climatiques supplémentaires qui pourraient permettre l'utilisation des méthodes plus précises, notamment celle de Penman qui rend bien compte du processus physique réel. La fiabilité de la méthode de Thornthwaite dans notre étude se résume à la superficie de la zone d'investigation et au calcul du bilan hydrologique qui est mieux exprimé par celle-ci (BROCHET et GERBIER, 1974). Les principaux paramètres recherchés sont l'évapotranspiration potentielle (ETP), l'évapotranspiration réelle (ETR) et l'excédent du bilan (P-ETR). On calculera ensuite l'infiltration efficace (Ie) après avoir estimé le ruissellement moyen annuel. Ainsi, pour le calcul de l'ETP de chaque mois, Thornthwaite propose l'équation 3 suivante :

$$
\mathrm{ETP}=16\left(\frac{10 . \mathrm{t}}{\mathrm{I}}\right)^{\alpha} \mathrm{F}(\mathrm{m}, \lambda)
$$

avec

ETP : évapotranspiration potentielle mensuelle $(\mathrm{mm})$;

$\mathrm{t}$ : température mensuelle moyenne en degré Celsius;

$\alpha$ : fonction complexe de l'indice thermique $\left(\alpha=6,75 \cdot 10^{-7} \cdot I^{3}-7,71 \cdot 10^{-5} \cdot I^{2}+1,79 \cdot 10^{-2} \cdot I+0,49239\right)$;

I : Indice thermique annuel est calculé selon l'équation 4 :

$$
I=\sum_{i=0}^{12} i
$$

avec

$$
\begin{gathered}
\mathrm{i}=\left(\frac{\mathrm{t}}{5}\right)^{1,514} \quad \text { : indice thermique mensuel } \\
\mathrm{F}(\mathrm{m}, \lambda) \quad: \text { facteur correctif fonction du } \\
\text { mois } \mathrm{m} \text { et de la latitude } \lambda
\end{gathered}
$$

L'ETP mensuelle étant calculée, les valeurs de l'ETR et de l'excédent sont estimées selon les procédures suivantes :

$1^{\text {er }}$ cas : Pour P $>$ ETP (P : pluviométrie du mois)

alors $\mathrm{ETR}=\mathrm{ETP}$

$\mathrm{Si} P+\mathrm{R}_{\mathrm{i}-1}-\mathrm{ETR} \geq \mathrm{RFU}$; alors $\mathrm{R}_{\mathrm{i}}=\mathrm{RFU}, \Delta \mathrm{RFU}=\mathrm{R}_{\mathrm{i}}-\mathrm{R}_{\mathrm{i}-1}$ et $\mathrm{S}=\mathrm{P}+\mathrm{R}_{\mathrm{i}-1}-\mathrm{ETR}-\mathrm{RFU}$

Si $\mathrm{P}+\mathrm{R}_{\mathrm{i}-1}-\mathrm{ETR} \leq \mathrm{RFU}$; alors $\mathrm{R}_{\mathrm{i}}=\mathrm{P}+\mathrm{R}_{\mathrm{i}-1}-\mathrm{ETR}, \Delta \mathrm{RFU}=$ $\mathrm{Ri}-\mathrm{R}_{\mathrm{i}-1}$ et $\mathrm{S}=0$;

$2^{\mathrm{e}}$ cas : Pour $\mathrm{P}<\mathrm{ETP}$

Si $\mathrm{R}_{\mathrm{i}-1} \geq$ ETP $-\mathrm{P}$, alors ETR $=$ ETP, $\mathrm{R}_{\mathrm{i}}=\mathrm{P}+\mathrm{R}_{\mathrm{i}-1}-$ ETR, $\Delta \mathrm{RFU}=\mathrm{R}_{\mathrm{i}}-\mathrm{R}_{\mathrm{i}-1}$ et $\mathrm{S}=0$;

Si $\mathrm{R}_{\mathrm{i}-1} \leq$ ETP $-\mathrm{P}$, alors ETR $=\mathrm{P}+\mathrm{R}_{\mathrm{i}-1}, \mathrm{R}_{\mathrm{i}}=0, \Delta \mathrm{RFU}=$ $R_{i}-R_{i-1}$ et $S=0$. 
avec : RFU : Réserve facilement utilisable par les végétaux (la valeur maximale est fixée ici à $100 \mathrm{~mm}$, compte tenu de la nature argilo-sableuse des sols); $\mathrm{R}_{\mathrm{i}}$ : Réserve effective du mois en cours;

$\mathrm{R}_{\mathrm{i}-1}$ : Réserve effective du mois précédent; $\mathrm{S}$ : Surplus disponible pour l'écoulement de surface ou souterrain du mois en cours; $\triangle \mathrm{RFU}=$ Variation de la réserve.

Pour le calcul de la lame d'eau ruisselée, nous avons appliqué le modèle de TIXERONT (1964) amélioré plus tard par MOUELHI (2003). L'idée de base adoptée en proposant ce modèle est l'existence d'une certaine corrélation entre la pluviosité moyenne annuelle et le ruissellement moyen annuel pour une longue période d'observation, une trentaine d'années par exemple. On estime que ces corrélations existent quand on a affaire à des bassins aux sols normalement constitués, ni trop perméables (terrains karstiques ou dunaires), ni trop squelettiques (zones trop arides en particulier). La formule de ce modèle amélioré s'établit selon l'équation 5 ci-dessous :

$$
R=\left[P^{4}+(a E)^{4}\right]^{1 / 4}-a E
$$

$\mathrm{R}$ : lame d'eau annuelle ruisselée $(\mathrm{mm})$;

$\mathrm{P}$ : lame d'eau précipitée annuelle $(\mathrm{mm})$;

E : lame d'eau évapotranspirée (ETP) annuelle; et

a: coefficient correctif de l'évapotranspiration potentielle (ETP).

Les estimations des lames d'eau ruisselées annuellement sur les différentes périodes d'observation du changement climatique permettront de quantifier l'impact du changement climatique sur les eaux de surface. Une fois ETR et R calculés, les estimations des infiltrations efficaces (Ie) se déduisent de l'équation du bilan hydrologique (Équation 6) :

$$
\mathrm{Ie}=\mathrm{P}-(\mathrm{ETR}+\mathrm{R})
$$

\subsubsection{Coefficients de tarissement et volume d'eau mobilisés par les aquiferes}

En absence de précipitation, un cours d'eau est alimenté par les eaux souterraines de son bassin versant. Il en résulte que le tarissement représente le volume d'eau libéré par cette réserve régulatrice pendant la durée de la vidange en régime non influencé. À travers cette relation entre eau de surface et eau souterraine, il s'est avéré important de connaitre l'évolution des réserves en eaux souterraines pendant les éventuelles variations climatiques. Les variabilités du coefficient de tarissement et du volume d'eau mobilisé par les aquifères définis par MAILLET (1905) peuvent nous permettre de mettre en évidence cette évolution. Les formules des courbes de tarissement donnent une valeur approchée des réserves régulatrices et les facteurs hydrologiques de la zone étudiée. L'expression mathématique du tarissement s'écrit selon l'équation 7 :

$$
\mathrm{Q}_{\mathrm{t}}=\mathrm{Q}_{\mathrm{o}} \cdot \mathrm{e}^{-\mathrm{kt}}
$$

avec :

$\mathrm{Q}_{\mathrm{t}}$ : débit à l'instant $\mathrm{t}$, exprimé en $\mathrm{m}^{3} \bullet \mathrm{s}^{-1}$;

$\mathrm{Q}_{0}$ : débit initial, exprimé en $\mathrm{m}^{3} \bullet \mathrm{s}^{-1}$;

$\mathrm{k}$ : coefficient de tarissement $\left(\mathrm{j}^{-1}\right)$.

L'équation 8 du coefficient de tarissement de Maillet est la suivante :

$$
\frac{\mathrm{e}^{-\mathrm{kt}}}{\mathrm{k}}+\frac{\mathrm{V}}{\mathrm{Q}_{0}}-\frac{1}{\mathrm{k}}=0
$$

L'intégration de cette équation sur l'intervalle $[0,+\infty)$ donne le volume annuel mobilisé par l'ensemble des aquifères du bassin versant, dont l'expression mathématique est définie par l'équation 9:

$$
\text { Vmobilisé }=\int_{0}^{+\infty} \text { Qoe }^{-\mathrm{kt}} \mathrm{dt}=\frac{\mathrm{Qo}}{\mathrm{k}}
$$

Pour le calcul du coefficient de tarissement en région tropicale, on utilisera la détermination par dichotomie proposée par COULIBALY (1997). Dans la méthode dichotomique, la courbe annuelle de tarissement est l'expression de la vidange du réservoir souterrain. La quantité d'eau récupérée à la station de jaugeage correspond exclusivement aux volumes dynamiques mobilisés par l'ensemble des aquiferes du bassin. De façon pratique, on part du débit journalier le plus élevé de l'année, considéré comme débit initial $\mathrm{Q}_{0}$ et on reporte en abscisse les débits mesurés tous les dix jours, jusqu'à ce qu'ils remontent de façon significative. Le pas de temps de dix jours a été retenu, car ce pas de temps a donné de bons résultats avec COULIBALY (1997), SAVANÉ et al. (2001) et SAVANÉ et al. (2003), respectivement dans le bassin versant du fleuve Sassandra et sur le Drou à Man. Ainsi, le coefficient de tarissement et le volume mobilisé chaque année par les aquifères sur le bassin du N'Zi et de la Comoé seront estimés et représentés graphiquement pour analyser les fluctuations.

\section{RÉSULTATS ET DISCUSSION}

\subsection{Résultats}

\subsubsection{Les tests de détection de rupture}

Dans la région de Bongouanou, la valeur moyenne de la pluviométrie de 1920 à 2005 est égale à $1300 \mathrm{~mm}$. La pluie annuelle maximale (1 $893 \mathrm{~mm})$ a été observée en 1950 . La 
valeur minimale de la pluviométrie a été de $910 \mathrm{~mm}$ et a été observée en 1983. Le coefficient de variation est égal à $110 \%$. On remarque une forte dispersion de la pluviométrie autour de la valeur moyenne. Ces résultats statistiques témoignent de la grande variabilité des précipitations dans cette région.

Le résultat du test de Pettitt montre que la série pluviométrique présente une rupture en 1968. La rupture est identifiée avec un seuil de confiance de $99 \%$. Le graphe de la variable du test de Pettitt (Figure 4) présente aussi une rupture probable en 1951. Mais le résultat du test indique que la rupture est peu significative. En effet, le calcul des moyennes pluviométriques annuelles des séries 1920-1951 et 1952-1968 donne respectivement $1444,8 \mathrm{~mm}$ et $1428,3 \mathrm{~mm}$, soit une différence peu significative de $16,5 \mathrm{~mm}$. La discrimination d'une variabilité climatique n'est donc pas bien perceptible dans la série 1920-1968, malgré l'existence d'une rupture probable. Ainsi, on distingue vraisemblablement deux grandes périodes :

- une période humide de 1920 à 1968, durant laquelle les pluies sont abondantes. Ainsi, plus de $60 \%$ des précipitations annuelles sont supérieures à la moyenne 1920-2005;

- une période sèche ou déficitaire de 1969 à 2005 où les précipitations annuelles sont faibles, seulement 12,2\% des pluies sont supérieures à la moyenne 1920-2005.

Les indices pluviométriques (indice de Nicholson) permettent de dégager les grandes tendances dans une série chronologique. L'étude de la variabilité interannuelle des indices pluviométriques de la zone d'étude montre aussi deux grandes tendances de variabilité climatique (Figure 5). On a une période humide de 1920 à 1968 où les indices sont caractérisés par une alternance de valeurs positives et négatives. Les indices positifs caractérisent les pluies supérieures à la moyenne et sont plus représentés dans la période humide. La période déficitaire de 1969 à 2005 est dominée par une succession d'indices négatifs montrant que les pluies annuelles sont presque toutes faibles et inférieures à la moyenne.

Une étude des variations des températures moyennes annuelles des périodes 1920-1968 et 1969-2007 a été effectuée et représentée par les graphes de la figure 6. L'analyse de ces graphes montre que la courbe de la période 1969-2007 est au-dessus de la courbe de la période 1920-1968, excepté pour les mois d'août et novembre. Dans l'ensemble, la température a subi une hausse d'environ $0,7^{\circ} \mathrm{C}$, pour presque chaque mois de l'année après la rupture de 1968 . Le changement climatique au cours de la période 1920-2005 se manifeste donc à la fois par une diminution très sensible de la pluviométrie et par une augmentation de la température.

\subsubsection{Résultat du bilan hydrologique}

Pour évaluer l'impact du changement climatique sur les ressources en eau dans cette région tropicale, nous avons calculé les paramètres du bilan hydrologique mensuel et annuel avant et après la rupture de 1968. Les résultats sont consignés dans les tableaux 1, 2 et 3. L'analyse des tableaux montre que la pluie moyenne annuelle, qui était estimée à 1 436,6 mm avant la rupture, est passée à $1163,4 \mathrm{~mm}$ après la rupture, soit une baisse de $19 \%$. L'excédent ou la pluie efficace, qui était de $297,5 \mathrm{~mm}$ avant la rupture, est réduit à $180,3 \mathrm{~mm}$ après la rupture, soit une baisse de $39 \%$. Le ruissellement moyen

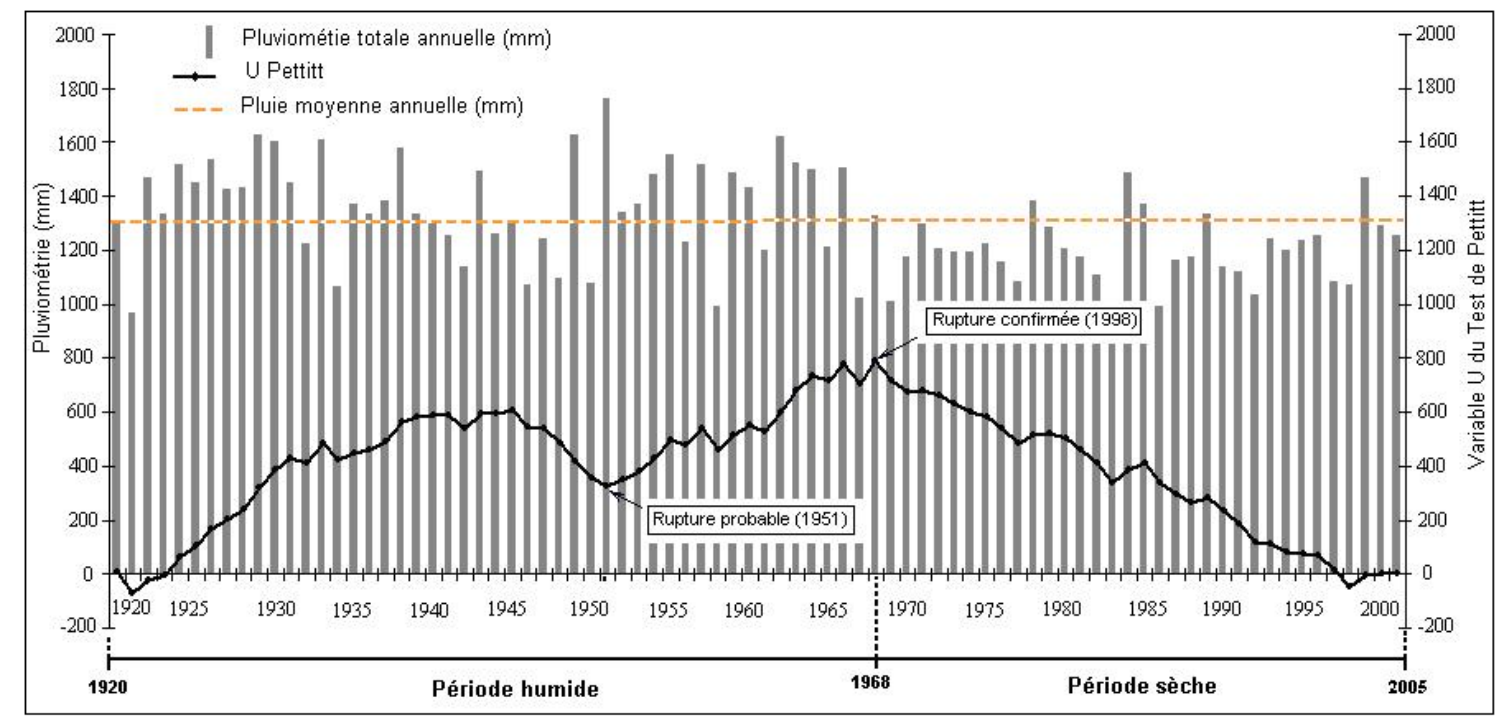

Figure 4. Fluctuations couplées de la pluviométrie et de la variable U du test de Pettitt (Station de Bongouanou). Coupled fuctuations of the rainfall and the variable $U$ of the Pettitt test (Bongouanou station). 


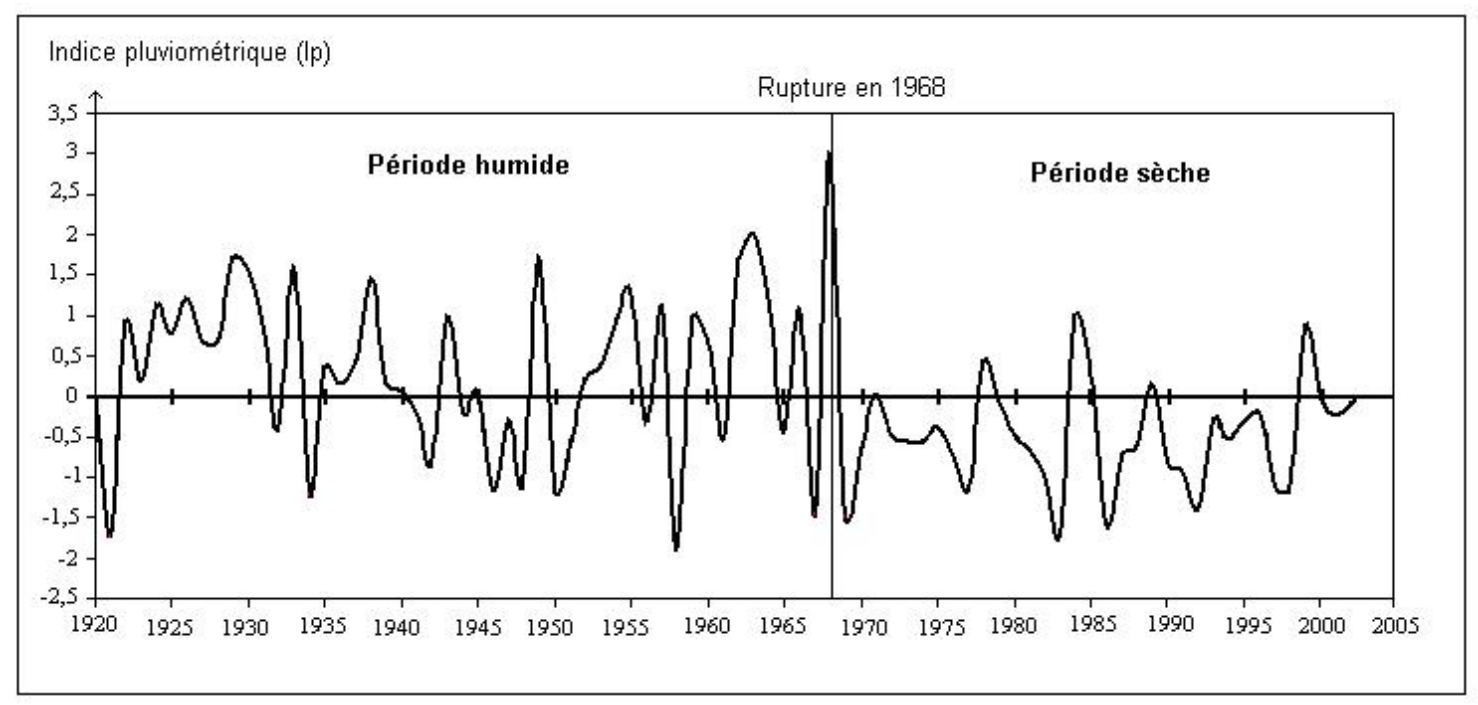

Figure 5. Fluctuation de l'indice de Nicholson de la zone d'étude (Station de Bongouanou). Fluctuation of the Nicholson index of the study region (Bongouanou station).

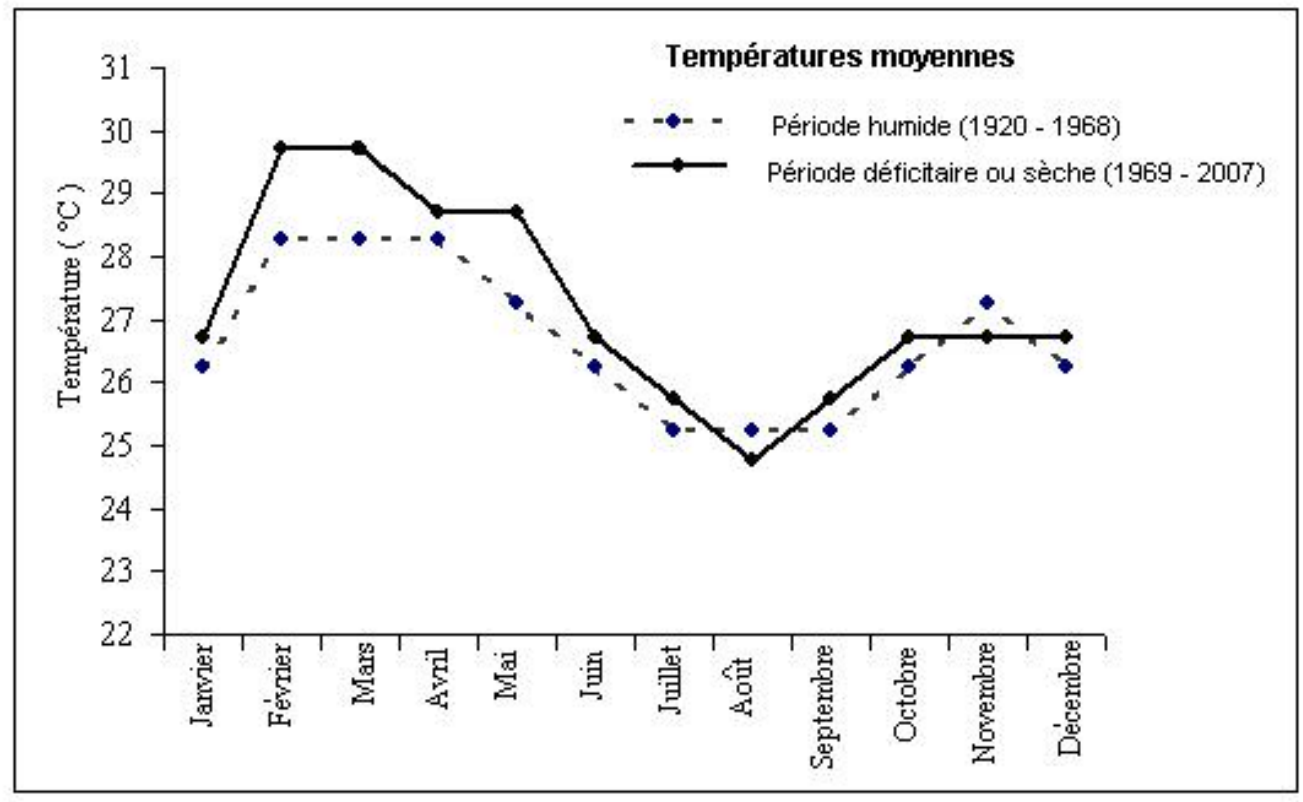

Figure 6. Variation des températures moyennes mensuelles autour de la rupture 1968 (Station de Bongouanou).

Variation of the average monthly temperature around the 1968 rupture (Bongouanou station

annuel, qui était environ $80 \mathrm{~mm}$ avant la rupture, est passé à $35 \mathrm{~mm}$ après la rupture, soit une baisse de $56 \%$. La lame d'eau estimée qui s'infiltre pour recharger les aquiferes de fissures de la région est passée de $217 \mathrm{~mm}$ avant la rupture à $145 \mathrm{~mm}$ après, soit une baisse de $33 \%$. On peut donc déduire de toutes ces observations que le volume d'eau qui s'infiltre dans les aquifères, qui était de $1,45 \mathrm{~km}^{3}$ en moyenne annuelle avant la rupture, est actuellement réduit à $0,96 \mathrm{~km}^{3}$. À la lumière de ces résultats, on peut dire que le changement climatique doit avoir entraîné une baisse non négligeable de la recharge des ressources en eau souterraine et, par conséquent, une diminution très sensible des écoulements, au moins en étiage, dans la région de Bongouanou. 
Tableau 1. Bilan mensuel par la méthode de Thornthwaite avant la rupture (1920-1968).

Table 1. Monthly water balance by the Thornthwaite method before the rupture (1920-1968.

\begin{tabular}{lccccccccccccc}
\hline \multicolumn{1}{c}{ MOIS } & Jan & Fév & Mars & Avr & Mai & Juin & Juil & Août & Sept & Oct & Nov & Déc & Total \\
\hline P (mm) & 20,1 & 26,2 & 92,1 & 190 & 231 & 247 & 193 & 60,2 & 80 & 159 & 102 & 36 & 1436,6 \\
ETP $(\mathrm{mm})$ & 82 & 81 & 105 & 105 & 117 & 116 & 108 & 107 & 107 & 128 & 108 & 90 & 1255 \\
ETR (mm) & 20,1 & 26,2 & 90 & 105 & 117 & 116 & 108 & 107 & 104 & 128 & 107 & 110 & 1139,1 \\
Surplus (mm) & 0 & 0 & 0 & 0 & 98,4 & 60,3 & 100,8 & 38,3 & 0 & 0 & 0 & 0 & 297,5 \\
Réserve du sol (mm) & 0 & 0 & 0 & 35 & 48 & 50,1 & 50 & 28,6 & 0 & 25 & 24 & 0 & \\
Variation de RFU & 0 & 0 & 0 & 35 & 60 & 30 & 0 & 0 & 0 & -15 & -45 & -65 & \\
ETP-ETR (mm) & 61,9 & 54,8 & 15 & 0 & 0 & 0 & 0 & 0 & 3 & 0 & 1,2 & -20 & 115,9 \\
P-ETR (mm) & 0 & 0 & 2,1 & 84,9 & 114 & 132 & 84,7 & -47 & -24 & 31 & $-4,9$ & -74 & 297,5 \\
\hline
\end{tabular}

Tableau 2. Bilan mensuel par la méthode de Thornthwaite après la rupture (1969-2005).

Table 2. Monthly water balance by the Thornthwaite method after the rupture (1969-2005).

\begin{tabular}{lccccccccccccc}
\hline \multicolumn{1}{c}{ MOIS } & Jan & Fév & Mars & Avr & Mai & Juin & Juil & Aoû & Sept & Oct & Nov & Déc & Total \\
\hline P (mm) & 17,3 & 28,1 & 93 & 142 & 195 & 213 & 121 & 50 & 61,4 & 145 & 78 & 20 & 1163,4 \\
ETP $(\mathrm{mm})$ & 92,2 & 91 & 102 & 103 & 116 & 102 & 102 & 107 & 116 & 106 & 121 & 115 & 1273,8 \\
ETR (mm) & 17,3 & 28,1 & 61,2 & 103 & 116 & 102 & 102 & 80,2 & 95 & 106 & 82,1 & 90 & 983,1 \\
Surplus (mm) & 0 & 0 & 0 & 0 & 60 & 67 & 45,2 & 8,1 & 0 & 0 & 0 & 0 & 180,3 \\
Réserve du sol (mm) & 0 & 0 & 0 & 26 & 48 & 38 & 10 & 21 & 40 & 22 & 12 & 0 & \\
Variation de RFU & 0 & 0 & 0 & 31 & 46 & 39 & 0 & 0 & 0 & -31 & -34 & -51 & \\
ETP-ETR (mm) & 74,9 & 62,9 & 40,8 & 0 & 0 & 0 & 0 & 26,9 & 21 & 0 & 39,2 & 25 & 290,7 \\
P-ETR (mm) & 0 & 0 & 31,8 & 38,9 & 78,8 & 110 & 18,5 & -30 & $-33,6$ & 40 & $-4,1$ & -70 & 180,3 \\
\hline
\end{tabular}

Tableau 3. Récapitulatif des paramètres du bilan hydrologique avant et après la rupture (1998).

Table 3. Summary of hydrological balance parameters before and after the rupture (1998).

\begin{tabular}{lc|c}
\hline \multicolumn{1}{c}{ Paramètres } & Avant la rupture (1920-1968) & Après la rupture (1968-2005) \\
\hline Précipitation (mm) & 1436,6 & 1163,4 \\
ETP (mm) & 1255 & 1273,8 \\
ETR (mm) & 1139,1 & 983,1 \\
Excédent (P-ETR) (mm) & 297,5 & 180,3 \\
Ruissellement (R) $(\mathrm{mm})$ & 80 & 35 \\
Infiltration efficace $(\mathrm{Ie})(\mathrm{mm})$ & 217 & 145 \\
\hline Volume d'eau infiltré $\left(\mathrm{km}^{3}\right)$ & 1,45 & 0,96 \\
\hline
\end{tabular}

\subsubsection{Résultat des calculs du tarissement}

Pour élucider d'avantagel'impact du changement climatique sur les réserves régulatrices de la zone d'étude, les coefficients de tarissement et les volumes mobilisés par les aquifères ont été déterminés pour chaque année de la période 1956-1985, avant et après la rupture de1968. Les résultats des calculs pour les fleuves N'Zi et Comoé ont été représentés graphiquement afin de mettre en évidence l'évolution des durées de vidange et les volumes mobilisés par les aquifères (Figure 7 et 8 ). De même, les valeurs moyennes de ces paramètres, avant et après la rupture, ont été estimées et consignées dans le tableau 4. Au niveau du fleuve N'Zi (Figure 7a), le coefficient de tarissement avant la rupture fluctue entre $0,015 \mathrm{j}^{-1}$ et $0,070 \mathrm{j}^{-1}$, avec une valeur moyenne de $0,036 \mathrm{j}^{-1}$ (inverse de 28 jours). Après la rupture, il varie entre $0,017 \mathrm{j}^{-1}$ et $0,080 \mathrm{j}^{-1}$, avec une moyenne de $0,046 \mathrm{j}^{-1}$ (inverse de 22 jours). Au niveau du fleuve Comoé (Figure $7 \mathrm{~b}$ ), le coefficient de tarissement avant la rupture fluctue entre $0,02 j^{-1}$ et $0,07 j^{-1}$, avec une moyenne de $0,04 j^{-1}$ (inverse de 25 jours). Après la rupture, il varie entre $0,020 \mathrm{j}^{-1}$ et $0,078 \mathrm{j}^{-1}$, avec une moyenne de $0,048 \mathrm{j}^{-1}$ (inverse de 20 jours). La tendance croissante des valeurs du coefficient de tarissement est bien marquée pour les deux courbes. Un raccourcissement de la durée de tarissement après 1968 est ainsi mis en évidence. Les volumes d'eau mobilisés par les aquifères au niveau du fleuve N'Zi (Figure 8a) fluctuent entre $0,70 \mathrm{~km}^{3}$ et $2,40 \mathrm{~km}^{3}$ avant la rupture de 1968 , avec une moyenne de $1,52 \mathrm{~km}^{3}$. Après la rupture, ces volumes varient entre $0,35 \mathrm{~km}^{3}$ et $1,50 \mathrm{~km}^{3}$ et leur moyenne est estimée à $1,07 \mathrm{~km}^{3}$. Au niveau du fleuve Comoé 


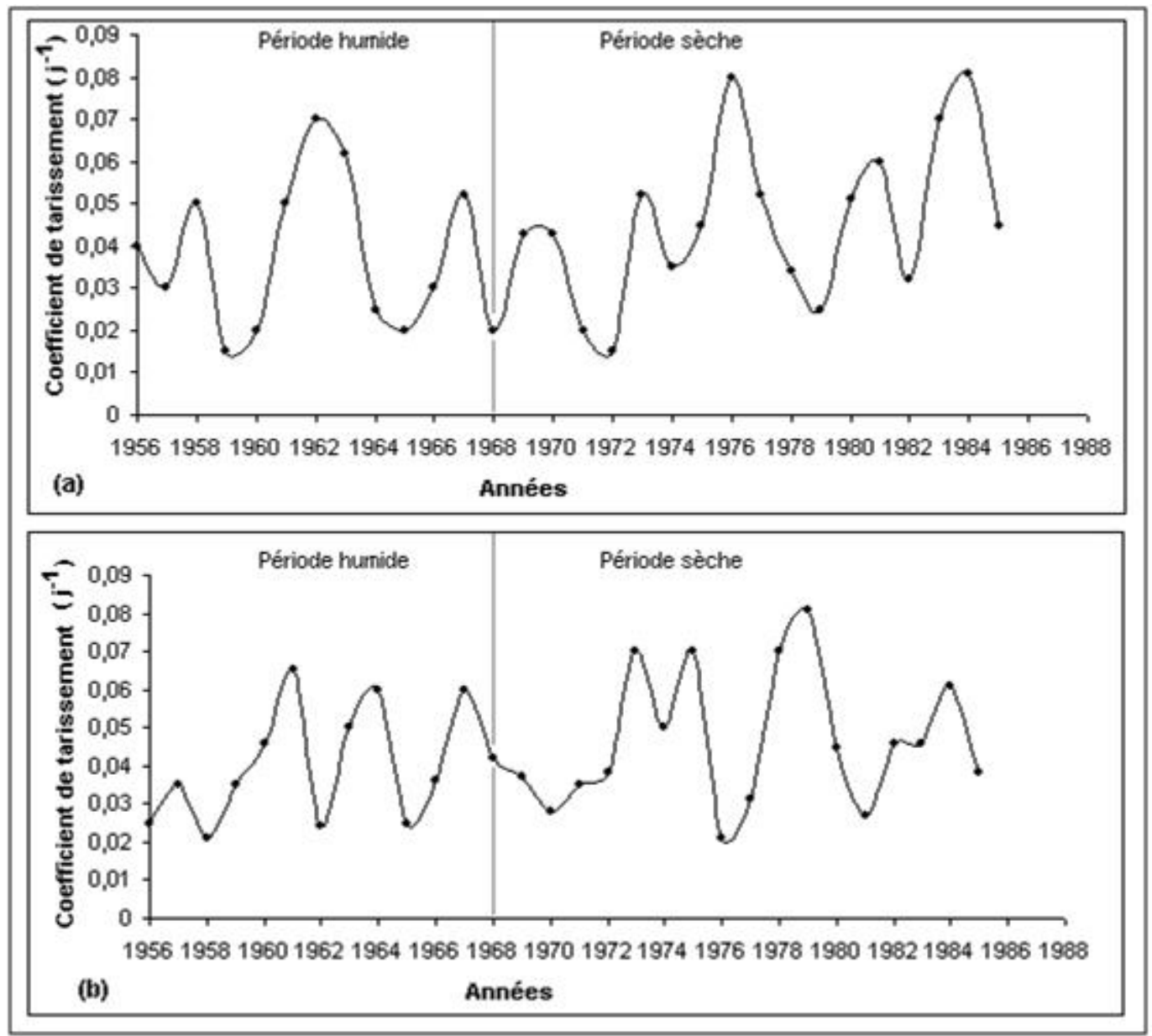

Figure 7. Variation des coefficients de tarissement dans la région de Bongouanou : (a) sur le N'Zi à la station de Bocanda; (b) sur la Comoé à la station Brou-Attakro. Variation of the recession coefficients in the Bongouanou region: (a) at Bocanda station, on the $N^{\prime} Z i$; (b) at Brou-attakro station, on the Comoé.

(Figure 8 b), le volume d'eau mobilisé avant la rupture fluctue entre $0,50 \mathrm{~km}^{3}$ et $2,30 \mathrm{~km}^{3}$, avec une moyenne de $1,43 \mathrm{~km}^{3}$. Après la rupture, ce volume d'eau varie entre $0,50 \mathrm{~km}^{3}$ et $1,45 \mathrm{~km}^{3}$ et la moyenne est $0,97 \mathrm{~km}^{3}$. De manière générale, on note dans la région de Bongouanou une tendance à la baisse d'environ $31 \%$ des volumes d'eau mobilisés par les aquifères après la rupture. Cette réduction des volumes d'eau mobilisés par les aquifères est interprétée comme la conséquence d'une moindre recharge qui se traduit en apparence par une vidange plus rapide. Après la rupture de 1968, on observe donc très régulièrement de plus faibles infiltrations efficaces chaque année, à cause de la réduction des précipitations. Ces résultats mettent en évidence une diminution des volumes d'eau mobilisés par les aquifères en période sèche ou déficitaire. Le changement climatique a donc entraîné, de manière générale, la réduction de la recharge des aquifères, et donc la baisse de la quantité d'eau souterraine dans les aquifères discontinus de la région de Bongouanou. La bonne concordance des résultats obtenus, au moyen de deux approches indépendantes (par l'évaluation de la recharge et par l'évaluation du drainage des aquifères) est tout particulièrement remarquable.

\subsection{Discussion}

La date de rupture obtenue pour la série pluviométrique de Bongouanou est 1968 selon les deux méthodes appliquées. L'année de rupture s'insère bien dans la période de rupture de la majorité des stations pluviométriques en Afrique de l'Ouest, particulièrement en Côte d'Ivoire, c'est-à-dire entre 1966 et 

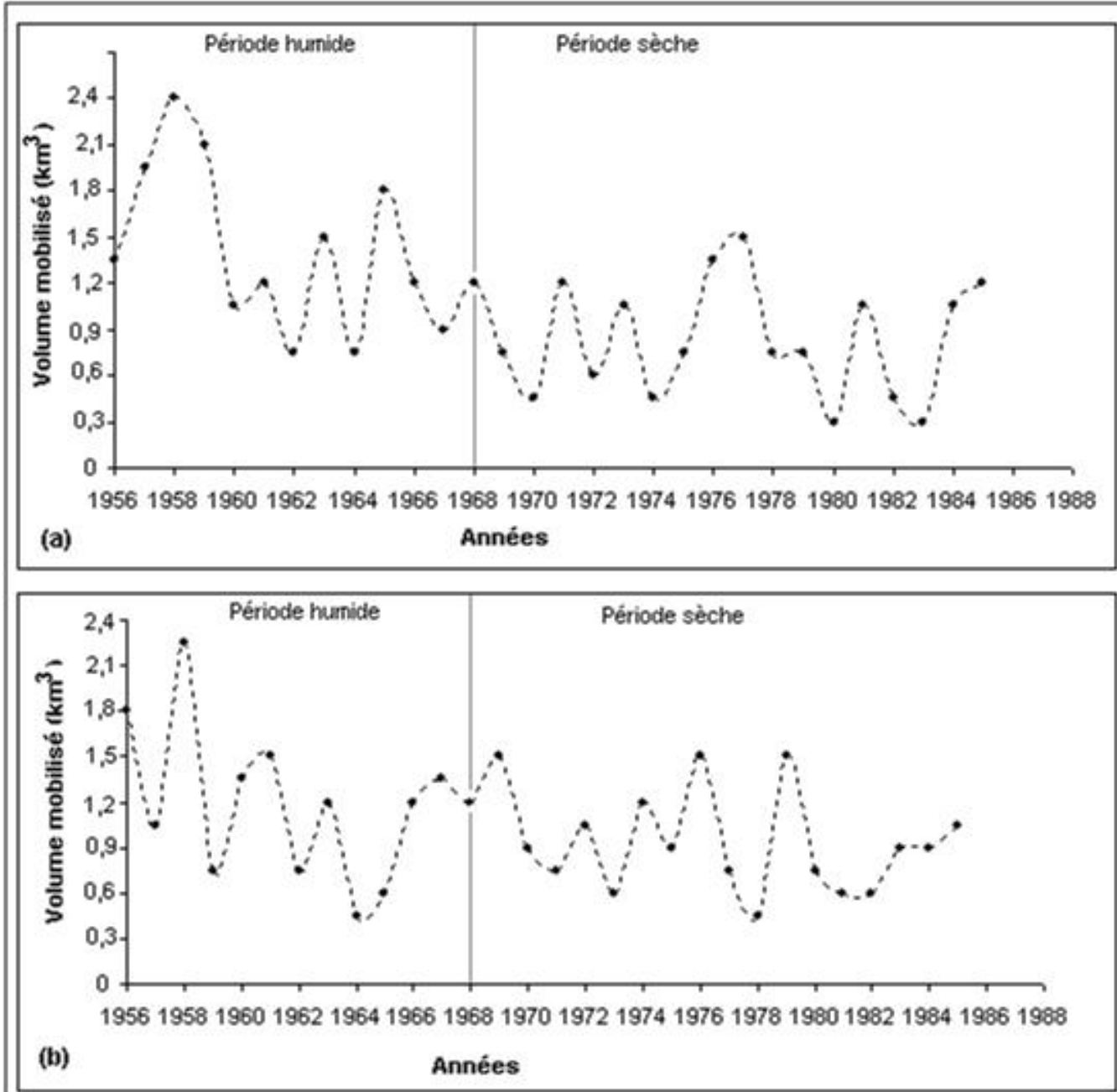

Figure 8. Variation des volumes d'eau mobilisés par les aquifères dans la région de Bongouanou : (a) sur le bassin du N'Zi; (b) sur le bassin de la Comoé.

Variation of the mobilized water volumes in the aquifers in the Bongouanou region: (a) in the N'Zi basin; (b) in the Comoe basin.

Tableau 4. Coefficients de tarissement et volumes d'eau mobilisés par les aquifères autour de la rupture 1968.

Table 4. Recession coefficients and mobilized water volumes in the aquifers around the 1968 rupture.

\begin{tabular}{lccc}
\hline \multicolumn{1}{c}{ Stations } & Périodes & Coefficients de tarissements $\left(\mathrm{j}^{-1}\right)$ & Volumes mobilisés $\left(\mathrm{km}^{3}\right)$ \\
\hline Bocanda & $1956-1968$ & 0,036 & 1,52 \\
$(\mathrm{~N} ' \mathrm{Zi})$ & $1969-1985$ & 0,046 & 1,07 \\
\hline Brou-Attakro & $1956-1968$ & 0,04 & 1,43 \\
$($ Comoé $)$ & $1969-1985$ & 0,048 & 0,97 \\
\hline
\end{tabular}


1972 (ADJA, 2009; ARDOIN, 2004; BROU, 1997; BROU et al., 1998; BROU, 2005; HUBERT et CARBONNEL, 1987; HUBERT et al., 1989; LUBÈS-NIEL et al., 1998; MARIEU, 1999; PATUREL et al., 1997; PATUREL et al., 1998; SERVAT et al., 1998; SIRCOULON, 1976). Le déficit pluviométrique qui en a résulté est en moyenne $19 \%$. Il est pratiquement de même ordre de grandeur que ceux obtenus par MORON (1996), SERVAT et al. (1998) et MEDDI et MEDDI (2009) dans les régions sahélo-soudaniennes. Cette similitude du comportement climatique serait d'échelle planétaire, car elle se vérifie également sur certaines zones du pacifique (GIEC, 2007; MERLE, 1995). Par ailleurs, ce changement des précipitations pourrait aussi être accentué par une augmentation de la température de l'air estimée à environ $0,7^{\circ} \mathrm{C}$ dans la région de Bongouanou et associée aux impacts des gaz à effet de serre (ADIAFFI, 2008), en accord avec une tendance à l'augmentation des températures des régions entre les deux hémisphères de l'ordre de $0,08{ }^{\circ} \mathrm{C} \bullet 10$ ans $^{-1}$, entrainant une dérégulation du mécanisme de migration du front intertropical (FIT), qui détermine le climat en Afrique de l'Ouest (ADIAFFI, 2008; GIEC, 2007; PÉNÉ et ASSA, 2003). Il ne peut aussi être exclu que la hausse des températures et la baisse de la pluviométrie soient aussi localement liées à la régression des forêts denses sempervirentes (effets liés à l'albédo et à une moindre évapotranspiration) (WWF, 2011), en plus, régionalement, de la variabilité de la dynamique de la circulation des masses d'air qui engendrent la mousson ouest-africaine (ADIAFFI, 2008).

Les cumuls des années déficitaires après 1968 ont eu pour conséquence une diminution très sensible de la recharge, entrainant une baisse régulière des volumes d'eau des aquifères de socle. Compte tenu de leur perméabilité et porosité relativement faibles, la capacité de stockage des eaux en milieu de socle est modeste et toujours sous la dépendance des fluctuations saisonnières et de l'intensité de la recharge (LACHASSAGNE et al., 2011; ROUX, 2005). La vidange rapide des cours d'eau de la plupart des bassins fluviaux en zones soudano-sahélienne et tropicale humide est due aux déficits pluviométriques observés depuis les décennies 1970 (BRICQUET et al., 1997; KANOHIN et al., 2009; MAHÉ et al., 1998; MAHÉ et al., 2000; OLIVRY et al., 1998). Néanmoins, certaines nappes phréatiques des bassins sédimentaires tertiaires du Niger ont enregistré une augmentation de leur recharge, malgré la réduction de la pluviométrique (FAVREAU, 2002; LEDUC et al., 2001), du fait d'une moindre évapotranspiration, et surtout d'une augmentation du ruissellement due au déboisement, avec une recharge plus forte, grâce à l'eau de surface accumulée dans les bas-fonds endoréiques. Dans la région d'étude, zone de socle, les effets du changement climatique (diminution de la recharge) semblent plus importants pour la ressource en eau que ceux liés à la déforestation.

\section{CONCLUSION}

Comme la plupart des pays de la zone tropicale, la Côte d'Ivoire est sujette au changement climatique. Cette étude menée dans sa partie Est de son socle, précisément le département de Bongouanou, illustre ce phénomène et donne une idée de son impact sur les ressources en eau. En effet, l'indice de Nicholson et le test de Pettitt indiquent une rupture en 1968 dans la série climatologique 1920-2005 et mettent en évidence une période humide, se situant entre 1920 et 1968, où les pluies sont abondantes, et une période déficitaire qui commence à partir de 1969 et se poursuit jusquaujourd'hui. La période déficitaire est caractérisée par une réduction de la pluviométrie annuelle d'environ $19 \%$ et une augmentation de la température de l'air estimée à $0,7^{\circ} \mathrm{C}$ pour presque tous les mois de l'année. Les ressources en eau ont subi une diminution non négligeable de leur alimentation suite à ce changement climatique, car le bilan hydrique montre une diminution de $56 \%$ des écoulements de surface et une baisse de $33 \%$ des infiltrations efficaces en moyenne annuelle après 1968. La diminution, du même ordre de grandeur, des tarissements des cours d'eau confirme cette estimation.

\section{REMERCIEMENTS}

Les auteurs adressent leurs remerciements aux correcteurs anonymes dont les commentaires ont permis d'améliorer le manuscrit.

\section{RÉFÉRENCES BIBLIOGRAPHIQUES}

ADIAFFI B. (2008). Apport de la géochimie isotopique, de l'hydrochimie et de la télédétection à la connaissance des aquiferes de la zone de contact "socle-bassin sédimentaire " du sud-est de la Côte d'Ivoire. Thèse de doctorat, Université Paris Sud, Paris, France, 230 p.

ADJA M. (2009). Étude de l'état hydrique saisonnier du bassin versant de la Bagoé dans un contexte de variabilité climatique. Départements de Boundiali et Tengréla (milieux soudanosahéliens au nord-ouest de la Côte d'Ivoire). Thèse unique, Université de Cocody, Abbidjan, Côte d'Ivoire, 170 p.

ARDOIN B. (2004). Variabilité hydroclimatique et impacts sur les ressources en eau de grands bassins hydrographiques en zone soudano-sahélienne. Thèse de doctorat, Université de Montpellier II, France, 330 p. 
BIGOT S., Y. BROU, J. OSZWAID et A. DIEDHIOU

(2005). Facteurs de la variabilité pluviométrique en Côte d'Ivoire et relations avec certaines modifications environnementales. Sécheresse, 16, 5-13.

BRICQUET J.P., F. BAMBA, G. MAHÉ, M. TOURE et J.C. OLIVRY (1997). Variabilité des ressources en eau de l'Afrique Atlantique, $P H I-V, 6,83-95$.

BROCHET P. et N. GERBIER (1974). L'évaporation : aspect agrométéorologique, évaluation pratique de l'évapotranspiration potentielle. Monographie Meteorol. Nat., 65, 95 p.

BROU Y. (1997). Analyse et dynamique de la pluviométrie dans le Sud forestier ivoirien : recherche de corrélation entre les variables climatiques et les variables liées aux activités anthropiques. Thèse de $3^{\mathrm{e}}$ cycle, Université de Cocody, Abidjan, Côte d'Ivoire, 211 p.

BROU Y. (2005). Climat, mutations socio-économiques et paysages en Côte d'Ivoire. Mémoire de synthèse des activités scientifiques présenté en vue de l'obtention de l'habilitation à diriger des recherches. Université des sciences et techniques de Lille, France, 212 p.

BROU Y., E. SERVAT et J. PATUREL (1998). Activités humaines et variabilité climatique : cas du sud forestier ivoirien. IAHS, 252, 365-373.

CANTAT O. (1995). Variabilité et variation climatiques en Normandie. L'évolution récente des températures à CaenCarpiquet. Sécheresse, 6, 273-279.

COULIBALY K.M. (1997). Evaluation du bilan hydrologique, de la variabilité climatique et du tarissement des cours d'eau par l'application de méthodes mathématiques dans le bassin versant du fleuve Sassandra (région de Buyo en Côte d'Ivoire). Mémoire de DEA en sciences de l'environnement, Université Abobo-Adjamé, Côte d'Ivoire, 80 p.

FAVREAU G. (2002). Le déboisement : origine d'une hausse de la recharge et des nitrates en aquifère libre semi-aride (Sahel, Niger). Pangea, 38, 25-34.

GIEC (GROUPE INTERGOUVERNEMENTAL D’ÉTUDE DU CLIMAT) (2007). Changements climatiques. Rapport de synthèse. http://www.ipcc.ch.

GUILLAUMET J.L. et E. ADJANOHOUN (1971). Milieu naturel de la Côte d'Ivoire. Végétation de la Côte d'Ivoire. ORSTOM, 50, 163-391.
HUBERT P. et J.P. CARBONNEL (1987). Approche statistique de l'aridification de l'Afrique de l'Ouest. J. Hydrol., 95, 165-183.

HUBERT P., J.P.CARBONNEL et A.CHAOUCHE (1989). Segmentation des séries : Application à des séries de précipitations et de débits de l'Afrique de l'Ouest. $J$. Hydrol., 110, 349-367.

KANOHIN F., M.B. SALEY et I. SAVANÉ (2009). Impacts de la variabilité climatique sur les ressources en eau et les activités humaines en zone tropicale humide : cas de la région de Daoukro en Côte d'Ivoire. Eur. J. Sci. Res., 26, 209-222.

KOUASSI A. (2008). Caractérisation d'une modification éventuelle de la relation pluie-débit et ses impacts sur les ressources en eau en Afrique de l'Ouest : cas du bassin versant du N'Zi (Bandama) en Côte d'Ivoire. Thèse unique de Doctorat, Université de Cocody, Abidjan, Côte d'Ivoire, 217 p.

LACHASSAGNE P., R. WYNS et B. DEWANDEL (2011). The fracture permeability of hard rock aquifers is due neither to tectonics, nor to unloading, but to weathering processes. Terra Nova, 23, 145-161.

LEDUC C., G. FAVREAU et P. SCHOETER (2001). Longterm rise en a sahelien water-table: the continental terminal in South-west Niger. J. Hydrol., 243, 43-54.

LENEUF N. (1959). L'altération des granites calco-alcalins et des granodiorites en Côte d'Ivoire forestière et les sols qui en sont dérivés. Doctorat ès Sciences Naturelles, Université de Paris, Paris, France, 210 p.

LUBÈS-NIEL H., J. MASSON, J. PATUREL et E. SERVAT (1998).Variabilité climatique et statistiques. Étude par simulation de la puissance et de la robustesse de quelques tests utilisés pour vérifier l'homogénéité de chroniques. Rev. Sci. Eau, 11, 383-408.

MAHÉ G. et J.C. OLIVRY (1995). Variations des précipitations et des écoulements en Afrique de l'Ouest et Centrale de 1951 à 1989. Sécheresse, 6, 109-117.

MAHÉ G., R. DESSOUASS, G. BANDIA et J.C. OLIVRY (1998). Comparaison des fluctuations interannuelles de piézométries, précipitations et débits sur le bassin versant du Bani à Douna au Mali. IAHS, 252, 289-295.

MAHÉ G., J.C. OLIVRY, R. DESSOUASSI, D. ORANGE, F. BAMBA et E. SERVAT (2000). Relation eaux de surface-eaux souterraines d'une rivière tropicale au Mali, 
C.R. Acad. Sci., Sciences de la Terre et des Planètes, 330, 689-692.

MAILLET E. (1905). Essai d'hydraulique souterraine et fluviale. HERMANN A. (Éditeur), Paris, 286 p.

MARIEU B. (1999). De différents aspects de la variabilité de la pluviométrie en Afrique de l'Ouest et Centrale non sahélienne. Rev. Sci. Eau, 12, 363-387.

MEDDI H. et M. MEDDI (2009). Variabilité des précipitations annuelles du nord-ouest de l'Algérie. Sécheresse, 20, 57-65.

MERLE J. (1995) Environnement climatique du Pacifique Sud. Dans : C.R. du collogue Environnement Climatique dans le Pacifique Sud, 28-31 mars, Paris, France. Fonds documentaire ORSTOM, Cote B*6629 17 p.

MORON V. (1996). Régionalisation et évolution des précipitations tropicales annuelles (1946-1992), Sécheresse, 7, 25-34.

MOUELHI C. (2003). Vers une chaîne cohérente de modèles pluie-débit conceptuels globaux aux pas de temps pluriannuel, annuel, mensuel et journaliers. Thèse de Doctorat, École nationale du génie rural, des eaux et forêts de Paris, France, $274 \mathrm{p}$.

NICHOLLS N. (1988). El Niño-Southern oscillation and rainfall variability. J. Clim., 1, 418-421.

NICHOLSON S.E. (1994). Recent rainfall fluctuation in Africa and their relationship to past conditions over the continent. Holocene, 4, 121-131.

OLIVRY J.C., J.P. BRICQUET et G. MAHÉ (1998). Variabilité de la puissance des crues des grands cours d'eau d'Afrique intertropicale et incidence de la baisse des écoulements de base au cours des dernières décennies. IAHS, 252, 189-197.

PATUREL J.E., E. SERVAT, B. KOUAME, H. LUBÈS-NIEL, J.M. MASSON, J.F. BOYER, M. TRAVAGLIOT et M. MATIEU (1997). Variabilité pluviométrique en Afrique humide le long du Golfe de Guinée. Approche régionale intégrée. $P H I-V, 16,1-31$.

PATUREL J., E. SERVAT et M. DELATTRE (1998). Analyse des séries pluviométriques de longue durée en Afrique de l'Ouest et Centrale non sahélienne dans un contexte de variabilité climatique. J. Sci. Hydrol., 43,937-945.
PÉNÉ B.C. et A.D. ASSA (2003). Variations interannuelles de la pluviométrie et de l'alimentation hydrique de la canne à sucre en Côte d'Ivoire. Sécheresse, 14, 43-52.

PENMAN H.L. (1948). Natural evaporation from open water, bare soil and grass. Proc. Roy. Soc., A1931032, 120-145.

PETTITT A.N. (1979). A non-parametric approach to the change-point problem. Appl. Stat., 28, 126-135.

ROUX J.C. (2005). Aquifere et eau souterraine en France. Chapitre1 "Généralités sur les eaux souterraines». Ouvrage collectif, Tome 1, Académie des sciences, BRGM-éditions, 41-51.

SAVANÉ I., K. COULIBALY et P. GIOAN (2001). Variabilité climatique et ressources en eaux souterraines dans la région semi-montagneuse de Man. Sécheresse, 12,231-237.

SAVANÉ I., K.M. COULIBALY et P. GION (2003). Étude comparative de trois méthodes de calcul du coefficient de tarissement des cours d'eau. Sécheresse, 14,37-42.

SERVAT E., J. PATUREL, H. LUBÈS-NIEL, B. KOUAME, J.M. MASSON, M. TRAVAGLIO, M. OUEDRAOGO et J. BOYER (1998). Identification, caractérisation et conséquences d'une variabilité hydrologique en Afrique de l'Ouest et Centrale. IAHS, 252, 323-337.

SIRCOULON J. (1976). Les données hydropluviométriques de la sécheresse récente en Afrique intertropicale. Comparaison avec les sécheresses de 1913 et 1940. Cahiers ORSTOM, Hydrologie, 13, 75-134.

SODEFOR (1922). Société de développement des plantations forestières. Rapport d'activité de l'année 1992-1993. SODEFOR, Abidjan, $61 \mathrm{p}$.

THORNTHWAITE C.W. (1948). An approach toward a rational classification of climate. Geogr. Rev., 38, 55-94.

TIXERONT J. (1964). Prévision des apports des cours d'eau : Dans : Symposium Eau de Surface, tenu à l'occasion de l'Assemblée générale de Berkely de L'UGGI, Berkely, CA, États-Unis, pp. 118-126.

TURC L. (1931). Évaluation des besoins en eau irrigation évapotranspiration potentielle. Ann. Agron., 12, 13-49.

WWF (2011). Les forêts et le climat à la croisée des chemins. Rapport forêts vivantes : chapitres 3, $32 \mathrm{p}$. 